\title{
Spatial discrimination learning by young rats
}

\author{
JEROME H. BLUE and SHERMAN ROSS \\ Laboratory of Developmental Psychology, National Institute of Mental Health \\ Bethesda, Maryland 20205, and \\ Howard University, Washington, D.C. 20059
}

\begin{abstract}
Twenty hooded rat pups were tested at 17 days of age on a left-right spatial discrimination task. One experiment used homing as the incentive $(n=10)$, and the other experiment used suckling as the incentive $(n=10)$. Each rat was given a maximum of 50 trials to reach the learning criterion. This was followed immediately by a reversal of the original spatial problem. The results showed that a large proportion of pups reached learning criterion with homing as an incentive. However, few pups reached criterion on the spatial reversal task using this incentive. When suckling was used as an incentive, half of the number of pups reached criterion on both the acquisition and reversal spatial tests.
\end{abstract}

Inappropriate behavioral measures for altricial animals have restricted the examination of learning in young rats. Recent evidence that olfactory capacity is developed in rats soon after birth (Teicher \& Blass, 1977) has provided new interest in the spatial discrimination behavior of young rats (Teicher \& Blass, 1977). Most studies have examined the spatial behavior of young rats by using a simple left-right spatial discrimination task, which is comparable to a stimulus-response situation (Olton \& Papas, 1979). This task has also been used to study spatial reversal behavior in rats. Spatial reversals are more complex than left-right spatial discriminations, since the animal must attend not to only one stimulusresponse situation, but to several. Spatial discrimination tasks have been reported as being appropriate for assessing the spatial behavior of young rats (Bulut \& Altman, 1974). To our knowledge, a direct comparison of the incentives used in these tasks under similar experimental conditions has not been done. Reports have indicated that homing is a good incentive because of the strong olfactory cues from the mother (Balut \& Altman, 1974). Kenny and Blass (1977) report that the opportunity to suckle is an incentive for spatial discrimination learning in young rats.

It is reasonable to expect that homing or suckling incentives could be used to examine simple and complex spatial discrimination learning. The purpose of this study was to compare the spatial discrimination behavior of young rats on the acquisition of a left-right spatial discrimination and its reversal, using homing (Experiment 1) and suckling (Experiment 2) as the incentive.

This study was carried out at Northeastern University, Boston, Massachusetts, prior to J. H. Blue's employment at NIMH. Thanks are due to Helen Mahut, Alex Skavenski, and Martin Block for helpful comments. Requests for reprints should be sent to Jerome H. Blue, National Institute of Mental Health, Laboratory of Developmental Psychology, 15-K, 9000 Rockville Pike, Bethesda, Maryland 20205.

\section{EXPERIMENT 1}

\section{Method}

Subjects. Ten male and female hooded rat pups, born in the rat colony of the Laboratory of Neuropsychology, Northeastern University, Boston, Massachusetts, were used. The mothers were impregnated at Blue Spruce Farms, Altamount, New York. A 12-h/12-h light/dark cycle was used.

Apparatus. The homing apparatus consisted of a small starting box, a choice area, a goal area, and a home cage, as described by Bulut and Altman (1974). The starting area $(9 \times 9 \times 7 \mathrm{~cm})$, through a small hole $(3.8 \mathrm{~cm}$ in diameter $)$, opened into a wooden two-choice discrimination area $(24 \mathrm{x}$ $30.9 \times 15 \mathrm{~cm}$ ) connected to the goalbox filled with shavings. A perforated partition blocked the entrance of the incorrect side. A " $T$ "-shaped partition divided the choice area into two sides, allowing entry into the goal area (Figure 1).

Procedure. Twenty-four hours before testing, all pups were removed from the mother. During this time, they were housed in a $31 \times 111 \mathrm{~cm}$ Plexiglas box. The floor was covered with wood shavings with an operating heating pad underneath. The animals were tested at 17 days of age, after $24 \mathrm{~h}$ of separation from the mother. Each animal was tested individually. Rats were arbitrarily assigned: five to the left and five to the right side as the correct path. Acquisition and reversal trials were

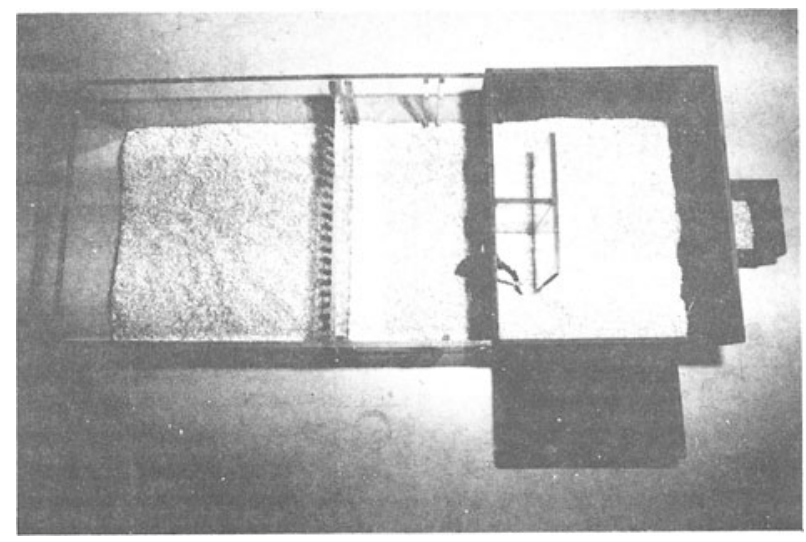

Figure 1. Homing apparatus (Bulut \& Altman, 1974). 
conducted on the same side until a learning criterion of 8 of 10 consecutive correct responses was reached. Each trial lasted a maximum of $60 \mathrm{sec}$. If no responses were made, the pups were placed in a closed wooden box, designated as the "isolation box" (Figure 1). Following an incorrect choice, pups were placed in the isolation box for $10 \mathrm{sec}$. Correct choices were followed by $15 \mathrm{sec}$ of reward. Reward was $15 \mathrm{sec}$ access to the goal compartment adjacent to the mother, allowing pups olfactory and auditory cues from her. The reversal test followed immediately after the acquisition task. Animals, who learned the task on the right, were rewarded only for going to the left and vice versa until $8 / 10$ consecutive correct responses were reached.

\section{Results}

Eight of the 10 pups reached criterion on the acquisition task when homing was used as an incentive. Only two of these eight pups reached criterion when they were required to reverse their learned habit (Table 1).

\section{EXPERIMENT 2}

\section{Method}

Subjects. Ten male and female hooded rat pups born and reared under the same conditions as in Experiment 1 were used in the experiment.

Apparatus. The suckling apparatus consisted of an anesthetized mother positioned on her side to expose her ventrum and nipple rows to the pups as they approached her from either side of the maze (Kenny \& Blass, 1977). The mother was placed in a Plexiglas compartment. The small starting box was suitable for infant pups. A partition divided the dam's surface into pectoral and inguinal goal compartments, each of two rows of three nipples. Nipple contact was thwarted in one compartment by wrapping that side of the entrance with a double layer of cheesecloth. This side was designated as nonreward. The reward side permitted the pups to suckle (Figure 2).

Procedure. The procedure was the same as in Experiment 1. The animals were tested at 17 days of age, after $24 \mathrm{~h}$ of separation from the mother.

\section{Results}

Five of the 10 pups reached criterion on the acquisition task, when suckling was used as an incentive. All pups that learned the acquisition task also reached criterion on the reversal task (Table 1).

\section{DISCUSSION}

The findings of this study suggest that both suckling and homing are effective incentives on tests of left-right spatial discriminations. When the same experimental conditions are used and young rats are required to reverse a spatially learned habit, suckling appears to continue as a sufficient incentive, but homing does not.

The results show that acquisition of a left-right spatial discrimination, which involves a simple stimulus-response situation, can be learned by young rats with either homing or suckling as an incentive. Spatial reversals, which are more complex
Table 1

Proportion of Pups Reaching Criterion on Acquisition and Reversal

\begin{tabular}{ccc} 
& \multicolumn{2}{c}{ Proportions } \\
\cline { 2 - 3 } Tasks & Acquisition & Reversal \\
\hline Homing & $80 \%$ & $25 \%$ \\
Suckling & $50 \%$ & $100 \%$ \\
\hline
\end{tabular}

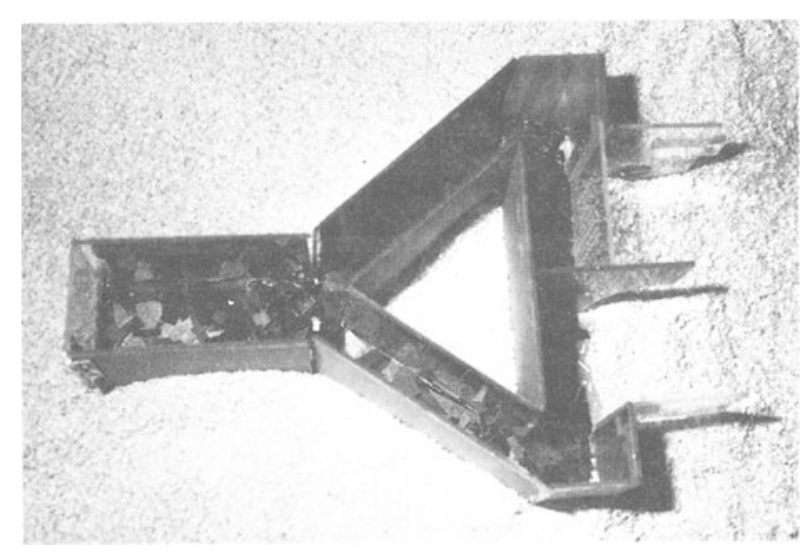

Figure 2. Suckling apparatus (Kenny \& Blass, 1977).

than tests of acquisition, are more easily learned by young rats when suckling is used. This finding might be related to the notion of resistance to extinction. Rat pups provided with an opportunity to suckle are more resistant to extinction (Capaldi, Myers, \& Davidson, 1981), unlike pups rewarded by homing, possibly due to the higher drive-producing effects associated with suckling.

In summary, both homing and suckling appear to be appropriate incentives to measure acquisition of simple left-right spatial discrimination in young rats. More complicated tasks, such as spatial reversals, require suckling incentives.

\section{REFERENCES}

Bulut, F. G., \& Altman, J. Spatial and tactile discrimination learning in infant rats motivated by homing. Developmental Psychobiology, 1974, 5, 465-473.

Capaldi, E. D., Myers, D. E., \& Davidson, T. L. A comparison of resistance to satiation and resistance to extinction. Animal Learning \& Behavior, 1981, 9, 108-114.

Kenny, J. T., \& Blass, E. M. Suckling as an incentive to instrumental preweanling rats. Science, 1977, 196, 898-899.

Olton, D. S., \& PaPas, B. Spatial memory and hippocampal function. Neuropsychologia, 1979, 17, 669-682.

Teicher, M. H., \& Blass, E. M. First suckling response of the newborn albino rat: The roles of olfaction and amniotic fluid. Science, 1977, 198, 635-636.

(Received for publication October 21, 1981.) 